\title{
Providers' and key opinion leaders' attitudes, beliefs, and practices regarding emergency contraception in Nigeria
}

\author{
Babatunde A.O. Ahonsi \\ Population Council \\ Salisu Mohammed Ishaku \\ Population Council \\ Araoyinbo Idowu \\ Population Council \\ Ayodeji Oginni \\ Population Council
}

Follow this and additional works at: https://knowledgecommons.popcouncil.org/departments_sbsr-rh

Part of the Gender and Sexuality Commons, International Public Health Commons, Maternal and Child Health Commons, Obstetrics and Gynecology Commons, Other Pharmacy and Pharmaceutical Sciences Commons, Public Health Education and Promotion Commons, and the Women's Health Commons How does access to this work benefit you? Let us know!

\section{Recommended Citation}

Ahonsi, Babatunde A. O. , Salisu Mohammed Ishaku, Araoyinbo Idowu, and Ayodeji Oginni. 2012.

"Providers' and key opinion leaders' attitudes, beliefs, and practices regarding emergency contraception in Nigeria," Final survey report. New York: Population Council. 
PROVIDERS'

AND KEY OPINION LEADERS'

ATTITUDES, BELIEFS,

AND PRACTICES

REGARDING EMERGENCY CONTRACEPTION

IN NIGERIA

BABATUNDE AHONSI, ISHAKU SALISU, ARAOYINBO IDOWU AND AYO OGINNI
FINAL

SURVEY

REPORT 
(C) 2012 The Population Council, Inc.

\section{(2) Population Council}

The Population Council confronts critical health and development issues-from stopping the spread of HIV to improving reproductive health and ensuring that young people lead full and productive lives. Through biomedical, social science, and public health research in 50 countries, we work with our partners to deliver solutions that lead to more effective policies, programs, and technologies that improve lives around the world. Established in 1952 and headquartered in New York, the Council is a nongovernmental, nonprofit organization governed by an international board of trustees.

\section{www.popcouncil.org}




\section{Table of Contents}

Contents i

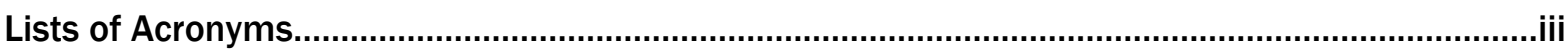

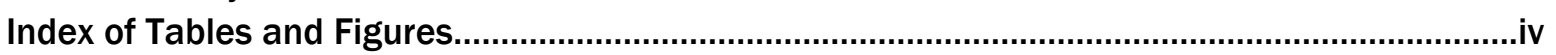

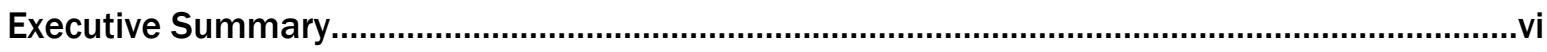

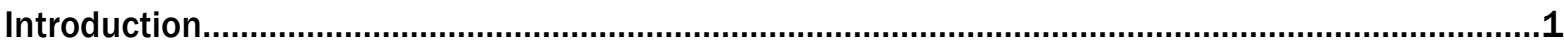

Review of the Literature....................................................................................................

Context and Rationale...........................................................................................................4

Methodology...........................................................................................................................4

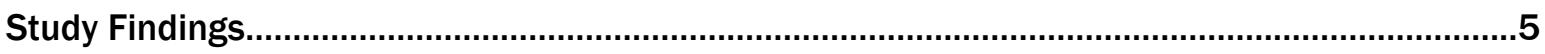

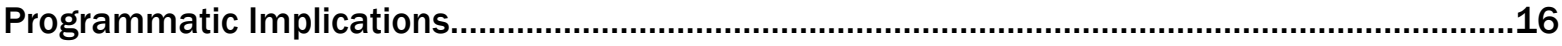

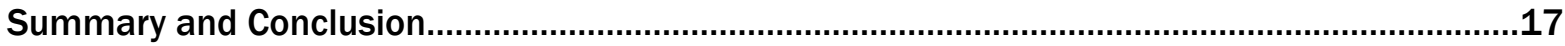

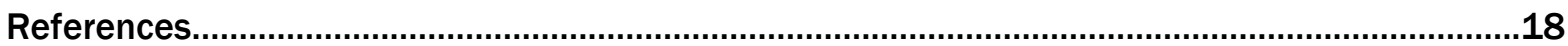


AOR

CSO

EC

ECP

FCDA

FMoH

FP

IDI

IRB

IUCD

KOL

$\mathrm{MCH}$

NAFDAC

NGO

NIMR

OtC

PMS

PMV

PPFN

RH

SDP

SFH

$\mathrm{SMOH}$

SOGON

STI

UNFPA

USAID
Adjusted odds ratio

Civil society organization

Emergency contraception

Emergency contraceptive pill

Federal Capital Development Authority

Federal Ministry of Health

Family planning

In-depth Interview

Institutional review board

Intra-uterine contraceptive device

Key opinion leader

Maternal and child health

National Agency for Food and Drug Administration and Control Non-governmental organization

Nigerian Institute for Medical Research

Over the counter

Patent medical store

Patent medicine vendor

Planned Parenthood Federation of Nigeria

Reproductive health

Service delivery point

Society for Family Health

State Ministry of Health

Society of Gynecologists and Obstetricians of Nigeria

Sexually transmissible infection

United Nations Fund for Population Activities

United States Agency for International Development 


\section{Index of Tables and Figures}

Table 1: $\quad$ Providers' understanding of EC in Nigeria based on selected variables

Table 2: $\quad$ Negative attitudes of EC providers in Nigeria

Table 3: $\quad$ Factors associated with providers' attitudes and beliefs about EC's safety

Table 4: $\quad$ Providers attitudes and beliefs about EC

Table 5: $\quad$ Factors associated with providers' attitude and beliefs about the efficacy of EC for pregnancy prevention

Table 6: $\quad$ Profile of EC providers' clients

Table 7: Information and services provided by EC providers to clients

Figure 1: $\quad$ EC products provided by service delivery points and facilities 


\section{Executive Summary}

Emergency contraception (EC) affords the opportunity of avoiding an unintended pregnancy after unprotected sexual intercourse. In its most common form, oral contraceptive pills, EC provides protection for up to 120 hours after intercourse. Alternatively, an intra-uterine contraceptive device (IUCD) can be inserted, no later than five or six days after intercourse. IUCDs have proven effective and, by expanding contraceptive choices, have transformed the sexual and reproductive lives of millions of women around the world. Despite significant progress in product development, procurement, and distribution in the past few decades, EC's capacity to benefit many women, in countries such as Nigeria, is hampered by several issues, including EC knowledge, attitudes, and practices among providers and opinion leaders, all of which are perceived as potential barriers to EC, but are not well understood.

To better understand these issues, The Population Council, with support from the International Consortium for Emergency Contraception, documented EC-related knowledge, attitudes, and practices among EC providers in Nigeria. Recognizing that providers do not operate in a vacuum, this study also assessed perceptions of key opinion leaders (KOLs) who help shape the policy environment for EC and family planning (FP) in general. The study is a part of a multi-country project also including India and Senegal.

Findings suggest significant gaps in provider EC knowledge, attitudes, and practices in Nigeria, potentially necessitating continuous training and re-training to help dispel misperceptions and negative attitudes. For example, many providers will not distribute EC to young, unmarried women, and clear instructions or counseling are rarely included when EC is provided. Moreover, almost all sampled providers were not aware that dedicated EC pills can provide protection for as many as five days postintercourse, despite its categorical statement in the country's FP service protocols.

Sampled KOLs demonstrated enthusiasm for supporting the method, but the Nigerian Government and its officials must support endorsement with action. While key policy stakeholders' attitudes towards EC are favorable, its procurement, distribution, and monitoring systems and procedures are currently almost entirely managed by non-governmental organizations (NGOs) and the private sector. In Nigeria, where much of the health infrastructure, facilities, and human resources are controlled by government, the public sector must play a greater role in providing EC-supportive policies and practices, and in monitoring their implementation. 


\section{Introduction}

Studies have shown a high proportion of all pregnancies in sub-Saharan Africa are unintended (Cleland et al. 2011). As a result, many end in termination through induced abortion. In countries such as Nigeria, where abortion law is highly restrictive, termination is commonly performed by unqualified persons, or in places not meeting required sanitary and technical standards, thereby propagating unsafe abortion, an important contributor to unacceptably high maternal mortality and morbidity ratios in many developing countries (Population Council and PSI 2009). Unsafe abortion remains a serious challenge to Nigeria's health system, a country with a maternal mortality ratio (MMR) estimated at 545 maternal deaths per 100,000 live births (NPC 2009). Unsafe abortion, as a result of unintended pregnancies, is identified as one of the leading causes of maternal deaths in Nigeria (Ebuehi et al. 2006).

With stringent legal and social restrictions to safe abortion in Nigeria, EC could play an important role in preventing unintended pregnancies, among both married and unmarried women who have had unprotected intercourse. EC is safe and effective in preventing unintended pregnancy, by either inhibiting or delaying, before ovulation, ovarian egg release, or potentially preventing sperm and egg convergence, by affecting cervical mucus or spermal binding ability. Although Nigeria's Federal Ministry of Health National Family Planning Guidelines lists EC among other modern contraceptive methods as a nonprescription drug sold over the counter, EC use remains very low in Nigeria (FMoH 2010, NPC 2009).

Few studies in recent years, however, have investigated provider EC knowledge, attitudes, and practices in Nigeria, and to the best of our knowledge, no study has investigated KOLs' EC perceptions and attitudes. The knowledge, attitudes and practices of EC providers, who are key gatekeepers, must be investigated, with gaps therein addressed. Perceptions of policy makers who both shape the general FP commodity environment, including EC, and enforce regulations affecting provider practices, need to be assessed. These issues constitute the study's main focus.

\section{Review of the literature}

\section{Providers' EC knowledge}

A 1998 study by the Society for Family Health (SFH) demonstrated a paucity of EC knowledge among health care providers in Nigeria. Only 61 percent of interviewed providers across the country knew of an EC method; Postinor was the only pill specifically packaged for EC (Society of Family Health 1998). EC knowledge varied regionally: It was higher in the southern zone than in the north (SFH 1998). In the southwest region, where EC knowledge seems high, only 33 percent of providers knew any effective method, with wide rural-urban differences $(22.4 \%$ and $5.8 \%$ respectively). Local pharmacy personnel were most aware of effective contraception methods, while those at patent medical stores showed poorest understanding of effective EC methods (SFH 1998).

The SFH study also reported that 29 percent of providers expressed belief that EC could result in infertility. Prescription method inconsistency also prevailed, reflecting a lack of detailed knowledge. No providers interviewed knew Postinor's dosage, side effects, and mechanisms of action, and only five providers (of 443) correctly stated the timing, as an EC agent, of IUCD insertion (SFH 1998).

The knowledge and attitudes of other trained health professionals, such as doctors, nurses, pharmacists, and hospital administrators, have been studied by Adekunle et al. (2000) in Ibadan, and their findings were not significantly different. For example, only 35 percent of respondents were aware that combined estrogen/progestin or progestin-only pills can be used as EC, and only 27 percent were aware IUCDs could be used as EC. Similarly, Obionu (1998) found, in Enugu, southeast Nigeria, that only 39 percent 
of health professionals were aware of an EC pill, and fewer still (26\%) were aware of IUCDs' potential contraceptive use. More worryingly, 29 percent of the sample who knew of an EC product expressed the belief its use was associated with infertility.

Although recent studies suggest improvement in EC provider knowledge in Nigeria, the situation is still far from ideal. As recently as 2006, 90 percent of health providers in Lagos State knew of an EC method, and 75 percent knew it could prevent pregnancy, but only 50 percent knew correct timing of administration, and only 10 percent were able to recognize the drug, its side effects, and dosage (Deborah 2007, Ebuehi et al. 2006). Most alarmingly, only two thirds of providers who knew of an EC method were aware it is legal in Nigeria. Okonofua et al. (2009) reported, from a survey of private medical practitioners (mainly doctors) in four Nigerian geo-political zones, that, while 80 percent of respondents correctly described EC methods, only 14 percent reported correct brands and dosages of ECs currently available in the country, and fewer still knew the exact timing for EC use.

Fayemi et al. (2010) report that the preferred service delivery point for EC in Nigeria, especially among young women, is patent medicine vendors, but only 46 percent of these vendors could correctly describe an EC, in terms of pregnancy prevention use. Less than 10 percent of patent vendors said it is a drug used after sex, and only three percent could mention a brand name (Postinor).

In another study, about 80 percent of senior health professionals in Borno State (Northeast Nigeria) reported hearing of EC, with awareness lower among nurses (70\%). Even so, up to 10 percent of these respondents were not sure of the correct timing of administration (Geidam, Kullima and Saddiq 2009).

Table 1 summarizes selected findings from previous research on EC knowledge among providers.

Table 1: EC understanding among providers in Nigeria, based on selected variables

\begin{tabular}{|l|l|c|}
\hline Authors and location of research & \multicolumn{1}{|c|}{ Variable of interest } & Measure \\
\hline SFH, across Nigeria (1998) & Knowledge of existence of EC & $60 \%$ \\
\hline Obionu, Enugu (1998) & Belief that EC causes infertility & $29 \%$ \\
\hline Obionu, Enugu (1998) & Knowledge of IUCDs as EC & $26 \%$ \\
\hline SFH, across Nigeria (1998) & $\begin{array}{l}\text { Knowledge of side-effects/dosage and mechanism of } \\
\text { action of Postinor }\end{array}$ & $0 \%$ \\
\hline Adekunle et al., Ibadan (2000) & Knowledge of IUCDs as EC & $27 \%$ \\
\hline Adekunle et al., Ibadan (2000) & $\begin{array}{l}\text { Knowledge of progestin/oestrogen or progestin-only pills } \\
\text { as EC }\end{array}$ & $35 \%$ \\
\hline $\begin{array}{l}\text { Deborah, Lagos (2007) \& Ebuehi } \\
\text { et al., Lagos (2006) }\end{array}$ & $\begin{array}{l}\text { Recognition of EC pills and knowledge of side effects } \\
\text { and dosages }\end{array}$ & $10 \%$ \\
\hline $\begin{array}{l}\text { Deborah, Lagos (2007) \& Ebuehi } \\
\text { et al., Lagos (2006) }\end{array}$ & Knowledge of legality of EC in Nigeria & $66 \%$ \\
\hline $\begin{array}{l}\text { Geidam, Kullima \& Saddiq, Borno } \\
\text { (2009) }\end{array}$ & Not sure of correct timing of EC & $10 \%$ \\
\hline $\begin{array}{l}\text { Okonofua et al., across Nigeria } \\
\text { (2009) }\end{array}$ & Knowledge of available brands and correct dosages & $14 \%$ \\
\hline
\end{tabular}


It is clear there is a paucity of knowledge and awareness of EC among all cadres of health care providers in Nigeria. This fact may, partly, explain why EC use among potential users is lacking, with widespread confusion about the exact mode of EC's operation particularly notable.

\section{Providers' attitudes towards EC}

Previous studies of EC providers also report findings on attitudes. Society for Family Health (1998), for example, found about 80 percent of all providers in Nigeria approved of EC, across all regional, religious, ethnic, and gender categories. Among the minority of providers disapproving of EC, reasons included religious belief, adverse side effects, EC as abortificient, and its use encouraging immorality, especially by unmarried adolescents (Deborah 2007, Ebuehi et al. 2006, Adekunle et al. 2000). It is not surprising, therefore, the majority of doctors, nurses, and pharmacists interviewed in Ibadan identified sexual assault $(64 \%)$ as the only appropriate indication for EC (Adekunle et al. 2000). Table 2 describes documented common negative attitudes towards EC in Nigeria.

Table 2: Negative attitudes of EC providers in Nigeria

\begin{tabular}{|l|l|}
\hline Prevailing attitudes & Source \\
\hline Forbidden by religion & SFH Nigeria (1998) \\
\hline EC an arbotificient & SFH Nigeria (1998), Deborah (2007) and Ebuehi et al. (2006) \\
\hline EC encourages immorality & SFH Nigeria (1998) \\
\hline EC causes infertility & Adekunle et al. 2000 \\
\hline
\end{tabular}

\section{EC providers' practices}

Of the 735 highly trained medical professionals—physicians and pharmacists—surveyed by Adekunle et al. in 2000, only 16 percent had ever prescribed the combined pills as EC, only 11 percent had ever prescribed the progestin-only pill, and only eight percent had prescribed an IUCD for EC. While 23 percent of private providers (predominantly doctors) across Nigeria offer EC services in their clinics, only 36 percent of health care providers working in public sectors in Borno State ever prescribed EC (Okonofua et al. 2009, Geidam, Kullima and Sadiq 2009).

Fayemi et al. (2010) also found, of patent medicine vendors (PMVs) who had ever dispensed EC in Ibadan, over 79 percent were actively dispensing. For PMVs not dispensing EC, reasons included fear of harassment by State Ministry of Health $(\mathrm{SMoH})$ and police, religious beliefs, and fear of sanction from the National Drug Law Enforcement Agency (NDLEA) and National Agency for Food and Drug Administration and Control (NAFDAC); others include fear of side effects, low demand, or product stock-out.

The current study provides opportunity for better understanding EC provider attitudes in Nigeria in a range of issues, but particularly the question of repeated use, and describing changes that have potentially occurred in the intervening period. 


\section{Context and Rationale}

Nigeria is the most populous country in Africa, with a 2010 estimated population of 160 million (Federal Government of Nigeria 2011). With a growth rate of 2.8 percent, the country is expected to double its population in 25 years if the current total fertility rate (5.7) and other determinants remain the same (Population Reference Bureau 2010). Nigeria's maternal and infant mortality are among the highest in the world: 545 per 100,000 and 75 per 1000, respectively (NPC 2009). Contraceptive prevalence for modern FP methods is 10 percent, and much lower for EC (less than 1\%).

Within this demographic context, assessing FP providers' knowledge, attitudes, and practices, especially regarding EC, will improve understanding of and strengthening for the supporting environment for EC's supply and use. In addition, the perceptions of KOLs and policy makers, regulators of these environments, and important influencers of health-seeking behaviors, will be assessed. Once these issues become clearer, more definite programmatic recommendations could be made.

Two urban centers were purposively selected-Abuja and Kaduna, in central and northwest Nigeria, respectively. Abuja, the Federal Capital Territory, is home to all ethnic populations, with a total population of approximately 1.4 million. As the seat of federal government, Abuja attracts the young and energetic migrating there in search of employment and other opportunities. Regrettably, the majority of this population lives in sprawling slums, exposing them to poor and substandard housing, without clean water and basic sanitation, and with insecurity and poor health services.

Kaduna is traditionally the political center of northern Nigeria and, therefore, harbors a large number of migrants mostly residing in unplanned, poorly serviced settlements. The most recent census placed Kaduna's total population at nearly 800,000 (National Population Commission 2009a). Kaduna, as with the rest of the region, has health and development indices worse than national averages; for example, contraceptive prevalence for the Northwest region is 4.6 percent (NPC 2009b).

\section{Methodology}

This study is a cross-sectional survey of providers' and stakeholders' beliefs, attitudes, and practices concerning EC provision and use in Kaduna and Abuja. A semi-structured questionnaire was developed, pilot-tested, and utilized for interviewing both public and private sector providers. Various stakeholders and KOLs directly or indirectly involved in providing, or influencing, continued EC availability and use were also interviewed using an in-depth interview (IDI) guide.

\section{Provider survey}

A simple, random sample of providers drew from a sampling frame (from health authorities in each city) consisting of a list of PMVs, private and public FP clinics, and registered pharmacists in Kaduna and Abuja. From every clinic or hospital (private or public) selected, the FP service head was interviewed, and from every selected pharmacy and patent medical store the proprietor was interviewed. From these sampling frames, 735 providers were selected. A pre-survey questionnaire indentified 550 providers in the two cities currently providing, or who have ever provided, EC. The minimum sample size was 364 and, allowing for non-response of 10 percent, a total of 407 providers were interviewed. To eliminate data entry errors and reduce time for data entry, a scan-able questionnaire for data collection was used, with data entered centrally. Data were cleaned, and files were created in EPI-INFO and SPSS. A codebook and frequency distribution of the variables and a plan of analysis and outline report with dummy tables were developed prior to data analysis and report writing. 


\section{Key Opinion Leaders' interviews}

In Abuja, key stakeholders were purposively selected from members of the national legislative houses (i.e. chairs of the Senate and House of Representatives health committees); officials of the federal ministries of Health and Women's Affairs; heads of relevant professional associations such as the Nigeria Medical Association, Pharmacists' Council of Nigeria, and National Association of Nurses and Midwives; relevant pharmaceutical companies and leading social marketing organizations; religious leaders; and leaders of reproductive health $(\mathrm{RH})$ and women's rights-focused civil society organizations (CSOs). Thirteen of these key stakeholders were interviewed by Council staff, and their responses were recorded with tape recorders and written notes, transcribed verbatim, with content analysis based on four pre-determined domains (see below).

\section{Ethical considerations}

IRB approval was obtained from both the Nigerian Institute for Medical Research (NIMR) and the Health Research Committee of the Federal Capital Development Authority (FCDA). Each participant was informed of the research's nature and was also informed that everything they said would remain confidential, their names would not be recorded, no payment would be made for participation, they had the right to not answer any question, and that they could terminate the interview at any point if they felt uncomfortable. Signed consent was obtained from every participant.

\section{Study Findings}

\section{Providers' characteristics}

The average age of the 407 providers was 35 , with 56 percent male and 44 percent female, a reflection of the labor force's overall gender imbalance. Most providers interviewed were pharmacists $(36 \%)$, followed by PMVs (30\%), private sector hospital and clinic staff $(22 \%)$, and public sector hospital and clinic staff $(12 \%)$. The high proportion of pharmacists as EC providers indicates their gradual increase in EC dispensation, which may be a desirable development, since pharmacists are better qualified and more informed than PMVs about EC and modern contraception in general (SFH 1998).

\section{Providers' EC knowledge, attitudes, and practices}

In the 1990s, Postinor 2 was the only dedicated EC commodity available in Nigeria. This study revealed a broadening of the range of EC brands, indicated in Figure 1.

Figure 1: EC products provided by SDPs and facilities in 2011

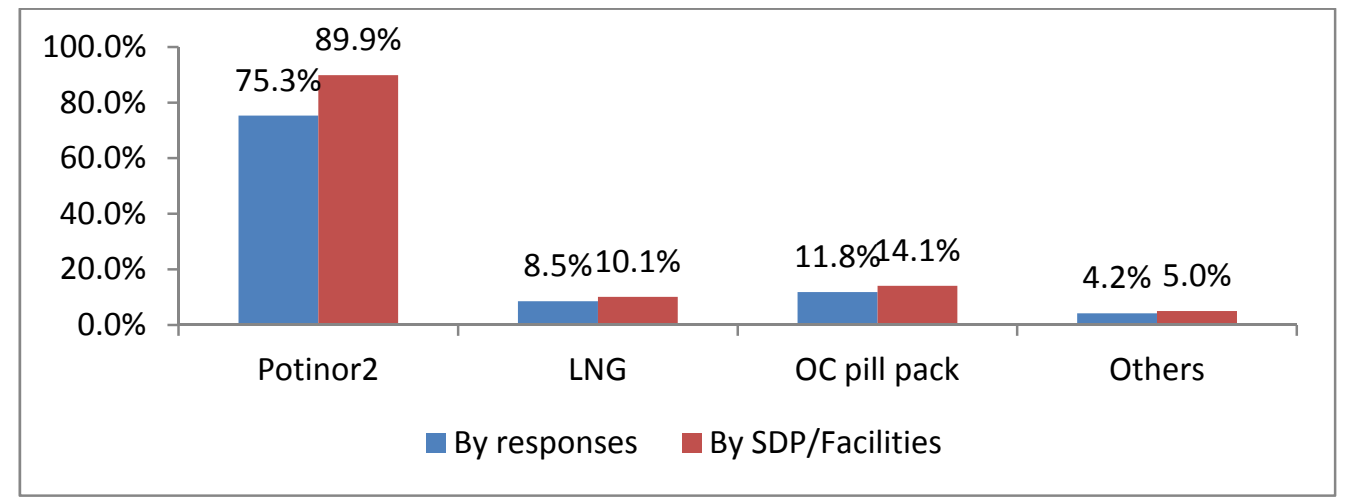


Of 407 providers interviewed, 125 (31\%) have been trained in FP counseling, and this is much higher for EC specifically (57\%). Some providers had obtained brochures or other forms of information around EC from the marketing representatives (39\%). The Federal Ministry of Health and women's health NGOs were not major sources of information for providers on EC (16\% and 3\% respectively).

Three-quarters of providers could correctly describe how EC works, which displays remarkable progress since the 1990s, when no interviewed providers were aware of how Postinor 2 works (SFH 1998). Ten percent of providers still consider EC an abortifacient, but which is an improvement compared with earlier studies, which found up to 32 percent of providers in Lagos thought EC is an abortifacient (Deborah 2007, Ebuehi et al. 2006). Only 29 percent of providers knew, however, that all women are eligible for EC. Remarkably, only one provider $(0.2 \%)$ was aware EC pills are protective for up to 120 hours after unprotected intercourse, despite current Nigerian EC service delivery guidelines clearly affirming this (Revised National Family Planning Service Protocols 2010).

Overall, only 12 percent of providers could be categorized as exhibiting comprehensive EC knowledge, knowing at least four of the five questions on EC knowledge. Factors significantly associated with comprehensive knowledge of how EC works included:

- $\quad$ Ever received FP counseling training, AOR 1.9 (CI1.02, 3.67)

- Ever received training on EC specifically, one year prior to survey AOR 1.54(CI 0.57, 4.18)

- Ever received a brochure/information document, AOR 1.67(CI 0.50, 5.23)

- Ever read an EC brochure/information document, AOR 1.91(CI 0.57, 6.69).

These patterns were also largely observed with other knowledge variables, as shown in Table 3.

Table 3: Factors associated with providers' attitudes and beliefs about EC safety

\begin{tabular}{|c|c|c|c|c|}
\hline Factors & $\begin{array}{c}\text { Total } \\
\text { N }\end{array}$ & $\begin{array}{c}\text { EC is safe to use } \\
N(\%)\end{array}$ & p-value & AOR (95\% Cl) \\
\hline \multicolumn{5}{|l|}{ Age } \\
\hline$<30$ & 114 & $106(93.0)$ & 0.594 & 1.00 \\
\hline$\geq 30$ & 251 & 237 (94.4) & & $1.25(0.48,3.24)$ \\
\hline Unknown & 42 & $38(90.5)$ & & $0.70(0.19,2.64)$ \\
\hline \multicolumn{5}{|l|}{ Gender } \\
\hline Male & 228 & $213(93.4)$ & 0.859 & 1.00 \\
\hline Female & 179 & 168 (93.9) & & $1.14(0.49,2.63)$ \\
\hline \multicolumn{5}{|c|}{ Ever received FP counseling training } \\
\hline Yes & 282 & $266(94.3)$ & 0.376 & $1.12(0.44,2.84)$ \\
\hline No & 125 & $115(92.0)$ & & 1.00 \\
\hline \multicolumn{5}{|c|}{ Ever received training on EC Specialty } \\
\hline Yes & 177 & $170(96.0)$ & 0.078 & $1.95(0.73,5.23)$ \\
\hline No & 230 & 211(91.7) & & 1.00 \\
\hline \multicolumn{5}{|c|}{ Received any brochure/information document } \\
\hline Yes & 165 & $156(94.5)$ & 0.525 & $4.63(0.23,93.30)$ \\
\hline No & 242 & 225 (93.0) & & 1.00 \\
\hline \multicolumn{5}{|c|}{ Read the brochure/information document } \\
\hline Yes & 149 & $140(94.0)$ & 0.827 & $0.21(0.01,4.23)$ \\
\hline No & 258 & $241(93.4)$ & & 1.00 \\
\hline \multicolumn{5}{|l|}{ SDP/Facilities } \\
\hline Public sector & 51 & 49 (96.1) & 0.896 & $1.57(0.29,8.42)$ \\
\hline Pharmacy & 146 & $136(93.2)$ & & $1.10(0.38,3.20)$ \\
\hline PMV & 122 & $114(93.4)$ & & $1.24(0.39,3.93)$ \\
\hline Private sector & 88 & $82(93.2)$ & & 1.00 \\
\hline
\end{tabular}




\begin{tabular}{lllcc}
\hline $\begin{array}{l}\text { Location } \\
\text { Kaduna } \\
\text { FCT }\end{array}$ & 204 & $191(93.6)$ & 0.990 & 1.00 \\
$\begin{array}{l}\text { SDP possessed guidelines on EC provision } \\
\quad \text { Yes }\end{array}$ & 203 & $190(93.6)$ & & $1.01(0.44,2.33)$ \\
$\quad$ & 268 & $254(94.8)$ & 0.182 & $1.44(0.62,3.34)$ \\
No & 139 & $127(91.4)$ & & 1.00 \\
\hline tSignificant p $<0.05$ & &
\end{tabular}

Table 4 illustrates Nigerian providers' negative attitudes about EC.

Table 4: Providers' attitudes and beliefs about EC use

\begin{tabular}{lll}
\hline \multicolumn{1}{c}{ Category } & \multicolumn{1}{c}{ Opinions } & $\%$ \\
\hline Safety & EC is safe to use & 93.6 \\
\hline Efficacy & EC is very effective for pregnancy prevention & 65.8 \\
\hline Access & Women should be provided with EC in advance of unprotected sex & 66.8 \\
& EC should be available OTC without prescription & 33.2 \\
\hline Frequency & There is no maximum number of times a woman should use EC in a year & 23.1 \\
of EC use & In one menstrual cycle, use EC each time after unprotected sex or each time it is needed & 17.7 \\
& There should be guidance/advice on frequency of EC provision for women & 89.9 \\
\hline Characteristics & Women who use EC repeatedly are not different from those who use once & 35.4 \\
of EC users & They are not more likely to participate in risky sexual behaviors & 30.0 \\
& They are not more likely to have more sexual partners & 35.6 \\
& They are not more likely to have STls & 22.1 \\
& They are more likely to use other contraceptive methods & 57.2 \\
& In the case of rape or sexual coercion & 49.6 \\
& Infrequent or unpredictable sex & 20.4 \\
& Not because of engaging in sex work & 100.0 \\
& Unprotected sex and wishing to use EC & 40.0 \\
& Married women & 55.5 \\
& In refugee or conflict settings & 11.8 \\
& Unmarried adolescent/young women & 14.0 \\
\hline & There should be no age restriction on EC use & 14.0 \\
\hline
\end{tabular}

Although 94 percent of providers expressed the belief that EC is safe, some negative attitudes were observed as widespread among EC providers. Over 34 percent of providers did not believe EC is very effective in preventing pregnancy, and two-thirds did not favor EC's general availability, over the counter (OTC) without a prescription. By implication, the subject of repeated use was not accepted by providers, as only 23 percent and 18 percent agreed to no limit on EC use and EC provision whenever needed, respectively. Fifty-seven percent of providers, however, had repeatedly provided EC to a client.

Ever received EC training and being a provider at a public facility are positively associated with perceiving EC as safe; AORs 1.95 (95\% CI 0.73, 5.23) and 1.57(95\% CI 0.29, 8.42) respectively. Likewise, ever having read a brochure/information document on EC is significantly associated with believing EC is effective in preventing pregnancies; AOR 3.66 (95\% CI 1.33, 10.08). Working at patent medical stores is strongly and positively indicative of positive attitudes toward providing EC to any woman who had unprotected intercourse. Table 5 (on page 13) indicates that belief in EC's efficacy for pregnancy prevention is strongly associated with prior exposure to specific information about EC. 
Table 5: Factors associated with providers' attitudes/beliefs about EC's efficacy for pregnancy prevention

\begin{tabular}{|c|c|c|c|c|}
\hline Factors & $\begin{array}{c}\text { Total } \\
\mathbf{N}\end{array}$ & $\begin{array}{c}\text { EC is very effective } \\
\text { for pregnancy prevention } \\
\mathbf{N}(\%)\end{array}$ & p-value & AOR (95\% Cl) \\
\hline \multicolumn{5}{|l|}{ Age } \\
\hline$<30$ & 114 & $75(65.8)$ & 0.896 & 1.00 \\
\hline$\geq 30$ & 251 & $164(65.3)$ & & $1.35(0.81,2.26)$ \\
\hline Unknown & 42 & $29(69.0)$ & & $1.68(0.75,3.78)$ \\
\hline \multicolumn{5}{|l|}{ Gender } \\
\hline Male & 228 & 161(70.6) & 0.022 & 1.00 \\
\hline Female & 179 & $107(59.8)$ & & $0.64(0.41,1.00)$ \\
\hline \multicolumn{5}{|c|}{ Ever received FP counseling training } \\
\hline Yes & 282 & 178(63.1) & 0.081 & $0.61(0.35,1.05)$ \\
\hline No & 125 & $90(72.0)$ & & 1.00 \\
\hline \multicolumn{5}{|c|}{ Ever received training on EC Specialty } \\
\hline Yes & 177 & $119(67.2)$ & 0.606 & $1.42(0.87,2.31)$ \\
\hline No & 230 & $149(64.8)$ & & 1.00 \\
\hline \multicolumn{5}{|c|}{$\begin{array}{l}\text { Received any brochure/information } \\
\text { document }\end{array}$} \\
\hline Yes & 165 & 105(63.6) & 0.437 & $\begin{array}{c}0.28(0.10 \\
0.75)^{\dagger}\end{array}$ \\
\hline No & 242 & $163(67.4)$ & & 1.00 \\
\hline \multicolumn{5}{|c|}{$\begin{array}{l}\text { Read the brochure/information } \\
\text { document }\end{array}$} \\
\hline Yes & 149 & $99(66.4)$ & 0.847 & $\begin{array}{c}3.66(1.33 \\
10.08)^{\dagger}\end{array}$ \\
\hline No & 258 & $169(65.5)$ & & 1.00 \\
\hline \multicolumn{5}{|l|}{ SDP/Facilities } \\
\hline Public sector & 51 & $24(47.1)$ & 0.001 & $0.70(0.34,1.45)$ \\
\hline Pharmacy & 146 & $105(71.9)$ & & $\begin{array}{c}1.99(1.12 \\
3.56)^{\dagger}\end{array}$ \\
\hline PMV & 122 & $89(73.0)$ & & $\begin{array}{c}2.20(1.17 \\
4.14)^{\dagger}\end{array}$ \\
\hline Private sector & 88 & $50(56.8)$ & & 1.00 \\
\hline \multicolumn{5}{|l|}{ Location } \\
\hline Kaduna & 204 & $122(59.8)$ & 0.010 & 1.00 \\
\hline FCT & 203 & $146(71.9)$ & & $1.53(0.98,2.38)$ \\
\hline \multicolumn{5}{|c|}{ SDP possessed guideline on EC provision } \\
\hline Yes & 268 & 185(69.0) & 0.060 & $\begin{array}{c}1.64(1.02 \\
2.63)^{\dagger}\end{array}$ \\
\hline No & 139 & $83(59.7)$ & & 1.00 \\
\hline
\end{tabular}


Table 6 describes the profile of EC users as reported by the providers. Most clients are predominantly students or unmarried $(60 \%)$ and married women $(54 \%)$; one quarter of providers indicated usually serving teenage adolescents (19 years or less).

Table 6: Profile of EC providers' clients

\begin{tabular}{ll}
\hline \multicolumn{1}{c}{ Dominant age profile } & \multicolumn{1}{c}{ N (\%) } \\
\hline Mostly teenage/adolescent clients (S19 years old) & $104(25.6)$ \\
Mostly student/unmarried clients & $245(60.2)$ \\
Mostly married women clients & $220(54.1)$ \\
Mostly men seeking EC for partners & $34(8.4)$ \\
\hline
\end{tabular}

The quality of information and services offered to clients by the providers was poor. Only 20 percent and 14 percent of providers reported offering information on side effects and responding to queries and concerns respectively. In addition, 49 percent of the providers provide EC without giving instructions to their clients (Table 7).

Table 7: Information and services to clients from EC providers

\begin{tabular}{lc}
\hline & N (\%) \\
\hline Provision of EC Pills & $105(25.8)$ \\
Provision of general information about EC & $199(48.9)$ \\
Provision of instructions on how to use EC & $208(51.1)$ \\
Provision of information on side-effects & $82(20.1)$ \\
Respond to queries and concerns & $58(14.3)$ \\
Refer to family planning services & $55(13.5)$ \\
\hline
\end{tabular}

Key Opinion Leaders' insights

Stakeholders' support will, to a large extent, determine supply and demand, legality and regulation, awareness of and support for, and public facility distribution, or lack thereof, of EC (Williams 2011). In some countries, EC availability and accessibility have stalled due to groups opposed to FP generally and those who perceive EC as abortifacient. EC's global availability and affordability, however, have increased over the last few years due multiple stakeholders' efforts (Schiappacasse and Diaz 2006). In some African countries such as Kenya, changing of policy support has affected commodity availability and stability. As contraception policy expands and contracts, contraceptive availability and accessibility also fluctuate, with RH repercussions in general (Crichton 2008).

KOLs' role in matters of public health importance cannot, therefore, be overstated. In Nigeria, such stakeholders' perceptions of EC have not been carefully investigated. This study addresses the perceptions of these Nigerian policy stakeholders in four areas: EC product knowledge; EC policy issues; supply and demand; and their recommendations for improving EC supply and utilization.

\section{KOLs' awareness of EC products in Nigeria}

In general, stakeholder knowledge of EC is encouraging. Government stakeholders, however, are apparently not directly involved in EC regulation and have not played a role in EC in the past: 
Their awareness of EC is mostly based on speculations and assumptions, with a few exceptions. For example, a federal legislator who reported never been involved or even informed of EC appears to have little understanding of what EC is, or indeed FP generally:

"Well, when you talk of EC, what I believe is that a contraception that is given to a woman, most of them are surgical contraception, in which a woman that is aborting, either induced or spontaneous, and she needs to be assisted."-Federal legislator

Even among those seemingly conversant with EC and its legality in the country, their responses were more conjectural than factual, indicating remoteness from FP policy generally, and of EC particularly. When asked about EC status and product knowledge in Nigeria, the following responses emerged:

"That is what I said. I don't have any information about [EC'S legal status]."

-Departmental deputy director, $\mathrm{FMoH}$

"As I am telling you ... Because we are not managing it [EC] in the public sector supply, I don't have detailed information [of available EC products], but I know the social marketing-SFH does that. So I don't know the brand, whether it is Postinor 2 or $1 . "$

-Departmental deputy director, FMoH

In contrast to public sector stakeholders, those working with NGOs and donor agencies seemingly understand the issue far better, which may reflect these different entities' roles in FP provision in general:

"Well ... legal status? Am not sure what you meant by that, but from my own understanding, do you mean if the drug is approved to be used in Nigeria? If that is what you mean ... the answer is Yes! And in fact, the two products being distributed by Society for Family Health actually are registered by NAFDAC, which is the highest drug approval body in Nigeria, that is ... Postinor 2 and Pregnor OCPs emergency contraceptives."

—Deputy director, leading health social marketing organization

"Of course ... from the Society for Family Health's experience ... we are battling with so much faking, particularly of the leading product ... Postinor 2 in Nigeria. In fact, you will see, if you go into the market, what they call Postinor 10, which you and I know is fake, because Gedeon Richter ... the manufacturer ... stopped producing Postinor 10 almost, like, 10 years ago. But if you still go into the Nigerian market you will still see it. Where are they getting it from? Nobody knows. I sincerely believe that what they are producing and distributing works for the clientele ... if you go to the market, you will still see various designs and shapes trying to imitate or copy what we are distributing. Of course, they are fakes and not duly registered by NAFD AC."

—Deputy director, leading health social marketing organization

"The legal status of emergency contraception in Nigeria ... as far as contraceptive methods are concerned, to the extent that they regulate fertility, it is very legal. There are a lot of policy statements from the government ... also protocols for the provision of such services, which are approved by the government ... So, to that extent, it is listed among contraceptives available for use in Nigeria. To that extent, that it is listed as one of the various means to regulate fertility, it is legal ... I cannot exactly pinpoint a particular law that says it is legal. But the point am making is ... to the extent that it is not probibited by the agencies that approve use of drugs in Nigeria ... to that extent, it is legal."

- Senior executive, national FP organization 
These examples illustrate the discrepancies in understanding among the two sectors. This is also observed in areas such as EC product effectiveness and safety. Again, most public sector stakeholder responses were conjectural as opposed to the firm knowledge and understanding demonstrated by stakeholders from NGOs and donor organizations. Only one participant, from SFH, however, was aware of counterfeit EC products in the Nigerian market. Most stakeholders, while acknowledging the possible existence of counterfeit EC, were largely based on assumptions:

"What I mean is that in any system you cannot expect 100 percent success or 100 percent pure provision of what is expected... definitely you will have some form of fake, and form of quackery and another form of contraband products which usually infiltrate into the system. So even if I don't believe, am sure it exists ... Yes!"--Senior executive, health sector regulatory agency

"As at the time I knew it in the "90s, there were no fakes, but now, I believe there are fakes for a product with a big market and consumption" —Departmental head, regulatory agency

"Not to my knowledge [- of EC counterfeit]."

— Head of medical team, Muslim women's NGO

Of the 13 stakeholders interviewed, less than half could describe how EC works. It is, however, instructive and encouraging that no respondents described EC as abortifacient, a potential predictor for a favorable EC policy environment in Nigeria, as the abortifacient belief may stall widespread EC adoption in many countries (Fayemi et al. 2010).

EC was generally perceived as appropriate for people who need it, and all stakeholders interviewed agreed there is dire EC need because of Nigeria's social, health, and economic circumstances. Concerns related to the possibility that widespread EC use could lead to lower barrier method use, with resultant escalation in sexually transmissible infections (STIs). Stakeholders' approvals of EC and concerns of likely STI transmission escalation include:

"I think it is a good product ... particularly where people have unpredictable sex. In Nigeria, a lot of us bave predictable sex and are in stable relationships, but it is excellent for young people whom, I bope, will use EC in addition to condoms." - - Head of medical team, Muslim women's NGO

'Well, the ones registered by NAFDAC are safe products! [giggles] So, I don't want to speak on generalization of the whole something ... the ones registered by $N A F D A C$ are safe and efficacious and fit for use by the members of the public, and I do recommend them to the members of the public... It should be in the health facilities, it should be in the pharmacies, patent medicine shops."

-Deputy director, public relations unit, health sector regulatory agency

"Yes, it should be encouraged; people should have varieties for them to be able to make an informed choice. That is it ... It should be available." -Deputy director, $\mathrm{FMoH}$

Only a few stakeholders were aware of any previous EC research in Nigeria. Almost all, however, were highly supportive of further EC research in Nigeria, believing that deeper understanding of how both EC clients and providers perceive EC will better inform programming for increasing its availability, accessibility, and use in Nigeria. Only one stakeholder, while acknowledging EC's importance, did not consider it worthy of further research.

"Well, I am not aware of any research on emergency contraception ... So, it goes to say that we actually need research on emergency contraception." - Federal legislator 
"Ehhmmm ... I personally have conducted some research at a stage ... collected some data on the awareness ... the knowledge ... and attitude on emergency contraception ... among adolescents ... Particularly youth corpers, and I am in the process of writing up ... but my observation is that not many people know about emergency contraception." - Senior executive, medical specialists association

"What are we doing research on EC for? When there are other areas of needs. We want to know ... how many of our health facilities are providing emergency obstetric care and all those things. We don't have resources to do that. The last [survey of facilities] ... was in 2002, and that's an obsolete document ... now is 2011_almost nine years!"-Departmental deputy director, FMoH

\section{Policy considerations for EC in Nigeria}

Most participants supported the view that EC should, as a provision of the National Family Planning Policy, be made widely available as part of the contraceptive mix. There was overwhelming enthusiasm among stakeholders for supporting EC's availability to the younger population at risk of unprotected intercourse and its potential consequences. While most interviewees were uncertain of whether national EC guidelines in Nigeria exist, EC's availability and widespread distribution were strongly recommended by all interviewed stakeholders, and undoubtedly positive signal for EC policy in Nigeria.

"Yeab I think it is better (EC) as a part of the (contraceptive) mix, and not singling it out to promote it, because when you promote it, you are promoting its use at the expense of regular use of contraception ... yeab! ... Young people in steady relationships would be able to use regular contraceptives."

- Head of medical team, Muslim Women's NGO

"Of course! Our belief is that if you go out there and see what some young women go through because they don't really know that there is something they can do immediately in the period post sexual intercourse. Some of them have gone into ... carrying pregnancies they never intended to, and in some instances it could lead to the termination of those pregnancies, and which also have some injurious impact to their health, or the health outcome may be detrimental. So I am strongly of the view that it should be part of the family planning regimen in Nigeria."-Deputy director, leading health social marketing institution

"Absolutely! It should be! It should be!!! Anytime, you teach contraception, emergency contraceptives should be the first because it's the one that prevents the deaths immediately."-Federal legislator

"I think it (EC) should be!! [in the national guidelines]. People may argue that the population of those who may need it may not be that significant, but I think if you really look closely, many young people coming with unwanted pregnancies, if they have had enough awareness, then you will know that many of these pregnancies could have been prevented through EC. And such population usually would not take routine family planning."-Senior executive, medical union

"EC should be part of the family planning program in Nigeria because the level of development and information available to users suggests the need for it. Prevention they say is better than cure, since other preventive methods are not readily available, EC is a quick intervention."

—Departmental head, health sector regulatory agency

'Providers like it! Based on the high demand for ECs in Nigeria, the patent medicine vendors are motivated to provide a wider range of services than the public sector, but they will need to be trained on the use of the product." - Program manager, bilateral development assistance agency

These examples illustrate EC's support and consideration within the Nigerian policy circle, from a diverse range of representatives. Participant responses did not suggest repeated use of EC is encouraged, however, as most stakeholders were unaware of repeated EC use in Nigeria and were not sure 
if such practices are associated with side effects. They did not particularly support repeated use. In addition, they did not readily perceive the health and social consequences of EC's unavailability when clients require it. Respondents supported, however, regular counseling and referrals of women tending to repeat use, for other more regular methods of contraception.

"What do you want [women] to do? The women are protecting themselves, repeat use could be dangerous since they don't know how to use them properly ... but women will use it."

-Chief technical officer, federal ministry

Another respondent stated:

'Like I said earlier, the class or category of clients that needs EC 'on a routine basis' is a peculiar group. This is a group that is not likely to buy into routine or conventional family planning ... ok! And yet they are sexually active and are naturally prone to pregnancies. These groups of young people, when they are aware of EC, do use them repeatedly. Is this a problem? Certainly it is in the sense that for such a group of individuals, it is safer in terms of protecting them from unwanted pregnancies if they would buy into routine family planning, but if you look at all the methods of routine family planning, they are not exactly fitted for most of them not just because of the safety and efficacy ... no!, but because of the secrecy associated before going into it. For this reason, they go for repeated EC use. Now, the unsafe nature of $E C$ is that, the more you use it repeatedly, the chances of its reduced efficacy is higher and therefore the risk. of getting pregnant, that is failure from using it is also bigher and the subsequent consequences of unwanted pregnancy. Beyond these, I don't see any problem associated with it."

-Senior executive, medical union

A minority view is represented by those who feel EC should be strictly regulated, supplied only after prescription, and that repeated use should not be tolerated:

"Yes, they do, and it is a problem. For youth, especially university students, for as many times they have sex, the drugs are used for fear of pregnancy. The males share the same view. There is need for research and the results used in the control of the drug. Therefore, usage should be restricted to prescription order which is filled-in by a registered pharmacy to ensure control along the distribution system. Emergency contraceptives are hormonal based, bence repeated use will create an imbalance in the system and the effects are better imagined."-Department head, federal regulatory agency

"That is where the abuse comes in.... when they just want to be using it instead of going on the regular pills ,„, and the regular ones are there, people have been trained, we have a lot of people trained and on all the methods." - Deputy director, federal ministry

\section{Service delivery, supply, and demand}

Respondents unanimously agreed that procurement of EC products in Nigeria is neglected by the government and is predominantly donor-driven, largely from one source. With Nigeria's large and growing population, increasing government participation in procuring EC and other FP commodities is seen as necessary by most interviewees. Even EC's marketing in Nigeria has been identified as polarized and monopolized, leading to poor satisfaction and cooperation among key stakeholders: 
"For as long as we keep the same supply and distribution of what we have, it's a buge challenge. We have only one source of public commodity-UNFPA. Only one social franchise-SFH. This is ridiculous for a country of over 150 million!! I can even understand the UNFP A thing, you understand because the source is pulled from a lot of places and they go to the manufacturers, but the social franchise involved in social marketing is only one group. Then, there is a problem because you don't challenge them; they do what they want to do, and you buy because you have no choice. I like SFH but I don't like monopoly" - Head of medical team, Muslim women's NGO

This observation is, in part, corroborated by another:

'Well ... first and foremost, if you look at problems of supplies within the pipeline ... the question is really there's no coordinated supply chain system for procurement in the country. Most procurement are done, not even by the national procurement system, but by individuals who think it is good business. So, you don't have any coordinated response and to that extent, we can't even quantify what we need. So the issue is that we don't even know the level of need for EC in the country. Because the procurement system is not coordinated, and it is not only to EC ...virtually in the last two years, we tried to come back to what we use to do in the '80s where there's a quantification process that leads to forecasting before you procure family planning contraceptives methods generally ... but in the mid '90s to 2000 that system collapsed because it is donor driven. So ... if you ask me ... we even have a defined pipeline system for $E C$ in the country? I'm afraid, no!" - Senior executive, national FP organization

All stakeholders interviewed shared the opinion that EC is appropriate for all categories of women who need it, but more so for young, unmarried women who have irregular and unpredictable intercourse and, therefore, do not require regular contraceptive methods. There was also consensus that all cadres of providers should be allowed to provide EC to clients, and that it should be non-prescriptive because of its emergency nature and the secrecy around its use. Stakeholders believed that restricting EC provision as a prescription drug, or only for provision by some categories of service providers in selected formal settings, will defeat the drug's emergency purpose:

"T believe all cadres of health care providers should provide or be involved in the prescription of EC. All the service delivery points should be involved in the provision of EC but a monitoring and regulatory system should be set up to effectively control the use of EC."

-Program manager, bilateral development assistance agency

Lack of awareness among clients on one hand, and lack of EC availability on the other, have been identified as the greatest barriers to EC use in Nigeria. Most respondents believe most women in Nigeria do not know EC exists, let alone know where to obtain it, complicated even further by the fact EC is not routinely available at convenient locations, or at a cost affordable to all. Some stakeholders opined that the lack of knowledge about EC among providers and preponderance of counterfeit drugs also contribute to access-related problems manifested in client dissatisfaction with the commodity. Inability to provide clients with adequate information on dosage, timing, and related issues such as side effects often leave clients confused, resulting in lack of use:

"So if you look at it from that perspective, I mean looking at your question, I would think that one big obstacle is sensitizing women on the availability, how to get it, when to use it and where to get it. Once we take care of these ... the barriers. I think the acceptability level would be high ..."

-Senior executive, national FP organization

"Availability, knowledge, accessibility are the common barriers to women accessing the product."

-Program manager, bilateral development assistance agency 
"One, once you recommend a drug that fails your client will lose confidence in you. The second one, is not knowing the appropriate regimen to take ... what to do in such a situation. Some will say hooo! give a start dose, then 12 hours later... but for us from experience, all of them work either immediately or 12 hours apart, but the issue is knowing what to do at a particular moment. And some say 'oooh! after 48 hours all hope is lost'... all hope is not lost, you can still help that woman in question. So, knowing what to do at a particular period, the effectiveness, how long it will last before it goes down is also very key." - Deputy director, leading health social marketing institution

\section{Recommendations}

Various suggestions and recommendations for improving EC in Nigeria were elicited from KOLs. Of particular importance is the Federal Ministry of Health' role, whose leadership, especially for FP generally and EC specifically, has diminished over the years, along with regulation and oversight, however the federal government is the sole body in Nigeria with required capacity and the infrastructure for catalyzing widespread EC use of EC:

"I think the key player, of course, is the Federal Ministry of Health, which is the center point of health policies in this country. But this should also trickle down to state and local government ministries of bealth because legislation for bealth in this country is on the concurrent list."

- Senior executive, medical union

Another recurring recommendation was the need for massive client and provider education and sensitizing them to EC's availability and use. Mass media was identified as key for improving the EC environment in Nigeria, as the majority of Nigeria's EC users receive their information from mass media.

"The first is to change behavior towards EC use and that I will do through massive enlightenment using the mass media. The second, it is to ensure that providers are well trained to the extent that they can counsel very well on EC provision. And the third, is to ensure that there's a coordinated pipeline system for EC in Nigeria." - Senior executive, national FP organization

Increasing participation of the federal, state and local governments, as well as enhancing provider and client understanding, are not all that are required for expanding EC access in Nigeria: Collaboration and participation by local pharmaceutical companies must also be sought. Their collaboration will ensure large-scale local production at an affordable price:

"And probably enable one of the indigenous pharmaceutical companies to go into production of something like levenorgesterone."-Senior executive, medical union

Further suggestions for improving EC services and sustaining quality involved continuous training, mentoring, and close supervision of service providers. Greater collaboration and networking between relevant authorities and regulatory bodies was recommended for ensuring strict quality control:

"Improve the knowledge of providers through regular training, mentoring, and supervision."

-Program manager, bilateral development assistance agency

"Well I think there is need for all sectors involved in this ... the manufacturers, the importers to rally round with regulatory agencies ... and then also see how they can put in place a kind of regulation that could promote the use of EC and widen the access and also remove hindrances through policy measures ... so I think it is a collective effort on the part of the policy makers, the providers, etc."

—Unit deputy director, regulatory agency 


\section{Programmatic Implications}

These research findings have several programmatic implications, both for providers and stakeholders. As less than half of EC providers in Nigeria have ever received training on EC, and as EC knowledge is directly related to such training, there is dire need in Nigeria for training providers on EC, especially those in the private sector. Training should particularly target PMVs and pharmacists who dominate EC sales ( $66 \%$ of the market combined).

The sale and purchase of EC is legal in Nigeria and is included in the FP and RH service delivery protocol. This is not enough. The service protocol is only visible seemingly on the bookshelves of the Federal Ministry of Health and is not widely distributed to, or read by, intended recipients. It is, indeed, quite striking that only one of 407 providers interviewed understood that EC protects against pregnancy for as many as 120 hours post-intercourse, while EC is included in the national service delivery protocol.

While EC knowledge and attitudes have improved substantially in recent years, negative perceptions are still rife among providers in Nigeria. Over 10 percent of surveyed providers still believe EC is an abortifacient, and the majority is averse to repeated use. Moreover, counseling by providers is not routine for EC clients. Consequently, FP and EC counseling training, EC brochures and information distribution, and national service delivery guideline distribution to all facilities must be given greater attention within FP policies and programs in Nigeria if EC demand and supply is to be significantly improved.

The Nigerian government needs to assume a leadership role in FP and EC commodity procurement and distribution, ensuring availability of high quality and effective products at prices young people can afford. Government officials must be cognizant of the changing realities of young people today. The strong support for EC expressed by interviewed government officials, backed by requisite action, will ensure EC is widely available within public sector facilities and that all of their providers are trained and wellinformed.

While escalating EC use through the public sector is important, the private sector's role must not be ignored; rather, synergy between the two sectors should be fostered. Since Nigeria's private sector is mainly interested in selling EC for profit and provides it to any woman who can buy it, a window of opportunity exists for EC advocates to work with this sector for improving EC supply and demand with, most importantly, improved skills for improved, quality service delivery.

\section{Summary and Conclusions}

EC is well-known in urban Nigeria, and is controlled by the largely unregulated and unmonitored private sector, with significant quality and client satisfaction implications. As government continues an indifferent role, FP and EC procurement and supply is almost entirely though donor agencies. This is not a sustainable situation. For EC availability and affordability to become standard and established, in both the private and public sectors, at quality and prices most users can afford, policy action is required, along with increased education for both clients and providers. Important information about EC that should be widely disseminated to both clients and providers includes its legality in Nigeria; its non-interference with established pregnancies; its safety, without adverse effects, for repeated use; its efficacy for as many as 120 hours following unprotected sex; and its suitability for all women, irrespective of age and marital status. 


\section{References}

Adekunle, AO, AO Arowojolu, AA Adedimeji and MA Okunlola. 2000. Emergency contraception: survey of knowledge, attitude and practice of health care professionals in Ibadan, Nigeria. $J$ Obstet Gynaecol 20(3): 284-289.

Antwi, KB. 2008. Stakeholders Perceptions of Current Health Education Situation under Ghana's Health Service. West African Journal of Applied Ecology 13(1): 83-95.

Cleland, JG, RP Ndugwa and EM Zulu. 2011. Family Planning in sub-Saharan Africa: Progress or Stagnation? Bull World Health Organ 89: 137-143.

Crichton, J. 2008. Changing Fortunes: Analysis of Fluctuating Policy Space for Family Planning in Kenya. Health Policy and Planning 23(5): 339-350

Deborah, K. 2007. Knowledge and Attitudes Toward Emergency Contraception Among Health Care Providers in Lagos, Nigeria. Journal of Community Medicine 3.

Drake, JK, H Espinoza, C Suraratdecha, Y Lacayo, BM Keith and JG Vail. 2011. Stakeholder perceptions of a total market approach to family planning in Nicaragua. Rev Panam Salud Publica 29(5): 329-336.

Ebuehi, OM, OAT Ebuehi and V Inem. 2006. Health Care Providers' Knowledge of, Attitudes Toward and Provision of Emergency Contraceptives In Lagos, Nigeria. International Family Planning Perspectives $32(2)$.

Fayemi, MM, OL Oduola, QC Ogbuji, K Osinowo, A Oyewo and OM Osiberu. 2010. The knowledge of emergency contraception and dispensing practices of Patent Medicine Vendors in South West Nigeria. Journal of Public Health Policy 31(3): 281-294.

Federal Ministry of Health, Nigeria. 2010. National Family Planning/Reproductive Health Service Protocols. Xxii $+302 \mathrm{pp}$.

Federal Government of Nigeria. 2011. 2011 Federal Budget. Abuja, Nigeria: National Budget Office.

Geidam, AD, AA Kullima and GU Saddiq. 2009. Knowledge, Attitude and Provision of Emergency Contraception among Health Professionals in Borno State Northern Nigeria. International Journal of Health Research 2(4): 339-346.

National Population Commission (NPC) [Nigeria] and ICF Macro. 2009. Nigeria Demographic and Health Survey 2008. Abuja, Nigeria: National Population Commission and ICF Macro.

Obionu, CN. 1998. Family Planning Knowledge, Attitude and Practice among Males in a Nigerian Population. East Afr Med J 75(3): 131-4.

Okonofua, FE, OO Lawrence, AH Afolabi and KA Osazee. 2009. A Survey of Knowledge and Practice of Emergency Contraception by Private Medical Practitioners in Nigeria. Journal of Chinese Clinical Medicine 4(1).

Population Council and Population Services International. 2009. Mainstreaming Emergency Contraception in Kenya: Final Project Report. Nairobi: Population Council and PSI.

Population Reference Bureau. 2010. World Population Data Sheet. Retrieved November 5, 2011, www.prb.org/pdf10/10wpds eng.pdf 
Schiappacasse, V and Diaz. 2006. Access to Emergency Contraception. Int. J of Obstetrics 94(3): 301-9. Epub July 12.

Society for Family Health. 1998. Emergency Contraception in Nigeria: Report of an Exploratory Research Project. Lagos: $\mathrm{SFH}$.

Williams, K. 2011. Provider-related Barriers to Accessing Emergency Contraception in Developing Countries: A Literature Review. Washington, DC: Population Council. 
